\title{
IMPROVEMENTS ON A UNIFIED DARK MATTER MODEL
}

A. Del Popolo ${ }^{1,2,3,4}$ and Xi-Guo Lee ${ }^{4}$

1 Dipartimento di Fisica e Astronomia, Universitá di Catania, Viale Andrea Doria 6, 95125 Catania, Italy

2 INFN sezione di Catania, via S. Sofia, 95123 Catania, Italy

3 International Institute of Physics, Universidade Federal do Rio Grande do Norte, 59012-970 Natal, Brazil

4 Institute of Modern Physics, Chinese Academy of Sciences, Post Office Box 31, Lanzhou 730000, Peoples Republic of China

\begin{abstract}
We study, by means of a spherical collapse model, the effect of shear, rotation, and baryons on a generalized Chaplygin gas $(\mathrm{gCg})$ dominated universe. We show that shear, rotation, and the presence of baryons slow down the collapse compared to the simple spherical collapse model. The slowing down in the growth of density perturbation is able to solve the instability of the unified dark matter (UDM) models described in previous papers (e.g. Sandvik et al. 2004) at the linear perturbation level, as also shown by a direct comparison of our model with previous results.
\end{abstract}

Key words: cosmology: theory, large scale structure of universe - galaxies: formation

\section{INTRODUCTION}

In recent years, analyses of data coming from Supernovae Type Ia (Amanullah et al. 2010), clusters of galaxies (Allen et al. 2008), and CMB (Spergel et al. 2007) lead to conclude that the geometry of our universe is flat, and that it is expanding at an accelerating rate due to an important but as yet not well understood component of the Universe, dubbed Dark Energy (DE).

In order to explain the quoted issues, several possibilities have been proposed. The simplest one is based on a non-zero value of the cosmological constant $\Lambda$ (for reviews, see Frieman et al. 2008; Li et al. 2011), usually interpreted as the vacuum energy density $\left(\rho_{\mathrm{v}}\right)$ which acts on the Friedmanns equations as a perfect fluid with negative pressure $\left(p_{\mathrm{v}}=-\rho_{\mathrm{v}}\right)$. The universe is then constituted not only of a material component whose existence is deduced from its gravitational effects, dubbed dark matter (DM), but also of a second dark component, namely DE. The resulting double dark cosmological model is the successful $\Lambda \mathrm{CDM}$ model, in which the overall cosmic fluid is constituted of non-relativistic matter (baryons + cold dark matter, $\left.\Omega_{\mathrm{nr}}=0.274\right)$ plus a vacuum energy density $\left(\Omega_{\Lambda}=0.726\right)$ fitting accurately the current observational data (Komatsu et al. 2011; Del Popolo 2007, 2013, 2014).

In the previous picture, one of the most challenging problems of $\Lambda \mathrm{CDM}$ cosmology is to understand the role played by the quoted cosmic components during 
the non-linear regime of gravitational clustering, and how they produce structures of different total mass such as galaxies and galaxy clusters (Del Popolo \& Gambera 1996, 2000; Del Popolo 1999, 2002; Hiotelis \& Del Popolo 2006, 2013) ${ }^{1}$.

Extensions of the $\Lambda \mathrm{CDM}$ model are based on the idea that DE is not related to the cosmological constant but, for example, related to a scalar field weakly interacting with matter (quintessence models) (Copeland et al. 2006) or known as K-essence, phantom models, or unified dark models (UDM) (e.g., Bertacca et al. 2010). In UDMs, DM and DE are described by the same physical entity. One peculiar case is the so-called generalized Chaplygin gas (gCg), introduced by Kamenshchik et al. (2001) and then developed in other studies.

The equation of state (EoS) describing the $\mathrm{gCg}$ is

$$
p=-\frac{C}{\rho^{\alpha}},
$$

where $C$ and $\alpha$ are positive constants, $\rho$ is the density, and $p$ is the pressure. When $\alpha=1$, the gCg corresponds to the standard Chaplygin gas $(\mathrm{sCg}){ }^{2}$.

The gCg background density evolution is

$$
\rho=\rho_{0}\left[\bar{C}+(1-\bar{C}) a^{-3(\alpha+1)}\right]^{\frac{1}{1+\alpha}},
$$

where $a$ is the cosmic scale factor, related to the cosmological redshift by $1+z=$ $a_{0} / a, \rho_{0}$ is the density at the present epoch, and $\bar{C}=C / \rho_{0}^{1+\alpha}$.

The EoS parameter, $w$, is given by

$$
w=-\bar{C}\left[\bar{C}+(1-\bar{C}) a^{-3(\alpha+1)}\right]^{-1} .
$$

It is important to stress that Eq. (3) shows that the gCg behaves as DM at early time $(a \rightarrow 0)$, and at later times $w \rightarrow-1$, approaching a $\mathrm{DE}$ behavior.

In the literature, several theoretical (Balakin et al. 2003) and observational consequences of the Chaplygin gas were studied. Cosmological tests were performed using CMB measurements (Amendola et al. 2003), measurements of X-ray luminosity of galaxy clusters (Zhu 2004), SNe Ia data (Bertolami et al. 2004), and lensing statistics (Dev et al. 2004).

In the context of UDMs with $\alpha \neq 0$, observations of large-scale structure (Avelino et al. 2004) and comparison of the linear theory with observations have put in evidence some problems of the gCg UDM. Avelino et al. (2004) studied the onset of the non-linear regime in gCg UDMs, showing that the transition from the DM behavior to the DE one is not smooth, and showed that, in gCg UDM, nonlinear effects generate a non-trivial backreaction in the background dynamics. This

\footnotetext{
${ }^{1}$ Despite its success the $\Lambda$ CDM model is plagued by a series of problems: (a) the small-scale problems, such as, e.g., the cusp/core problem (Cardone \& Del Popolo 2012; Del Popolo et al. 2013a; Del Popolo \& Hiotelis 2014), the missing satellite problem, and the too-big-to-fail problem (Del Popolo \& Gambera 1997; Del Popolo et al. 2014); (b) the cosmological constant problem (Weinberg 1989; Astashenok \& Del Popolo 2012), and the cosmic coincidence problem; (c) the debate on the universal nature of dark matter density profiles forming in the $\Lambda$ CDM cosmology (Navarro et al. 2010; Del Popolo 2010, 2011).

2 The sCg takes his name after Sergey A. Chaplygin, the Russian physicist who studied it in a hydrodynamical context (Chaplygin 1901).
} 
implies a breakdown of the linear theory at late times (even on large scales), for all $\alpha \neq 0$ models. They also pointed out the need to take into account non-linear effects when comparing with cosmological observations.

Sandvik et al. (2004), for example, found oscillations or exponential blowup of the dark matter power spectrum inconsistent with observations.

In order to have a clearer idea of the importance of the $\mathrm{gCg}$ as an alternative to the $\Lambda \mathrm{CDM}$, it is necessary to study the nonlinear evolution of DM and DE in the Chaplygin gas cosmology (Bilic et al. 2004). Moreover, a fully non-linear analysis would require SPH simulations (see Li et al. 2011). An alternative analytical approach to perform the quoted non-linear analysis and study the non-linear evolution of perturbations of DM and DE, is the popular spherical collapse model (SCM) introduced in the seminal paper of Gunn \& Gott (1972).

Fernandes et al. (2012) used the SCM to perform the quoted non-linear analysis. Their Friedmann-Lemaître-Robertson-Walker (FLRW) universe was endowed with two components: $\mathrm{gCg}$ and baryons ${ }^{3}$. An interesting feature of Fernandes et al.'s treatment is the fact that, differently from other works (e.g., Bilic et al. 2004; Pace et al. 2010), they considered the collapse of both gCg and baryons. Moreover, they assumed, for the background and the collapsing region, a timedependent equation-of-state parameter $w$, and derived a more accurate expression for the effective sound speed, $c_{\text {eff }}^{2}$, with respect to previous studies (e.g., Abramo et al. 2007). However, their study did not consider two important factors, namely, rotation (vorticity), $\omega$, and shear, $\sigma$. Nevertheless, in any proper extension of the $\mathrm{SCM}$, the contraction effect produced by shear and the expansion one produced by vorticity should be considered, as done by Engineer et al. (2000). The previous authors studied the effect of shear and vorticity only in DM dominated universe, and only in Del Popolo, Pace \& Lima (2013b,c) shear and vorticity effects were considered in the case of DM and DE dominated universes.

In the present paper, we study how the shear, $\sigma$, and the vorticity, $\omega$, change the results obtained by Fernandes et al. (2012). The paper is organized as follows. Section 2 summarizes the model used. It reviews the derivation of the equation of the SCM in the presence of shear and vorticity, the effective sound speed used, and the way the equations were integrated. Section 3 presents the results and Section 4 summarizes the conclusions.

\section{MODEL}

A popular analytical approach to study the non-linear evolution of perturbations of dark matter (in the presence of a non-clustered DE) is the standard SCM proposed in the seminal paper of Gunn \& Gott (1972) and extended in subsequent papers (Ryden \& Gunn 1987; Le Delliou \& Henriksen 2003; Basilakos et al. 2010; Del Popolo \& Kroupa 2009; Cardone et al. 2011a,b; Del Popolo 2012a,b.).

In the model, the overdensity is divided into mass shells, each one expanding with the Hubble flow from an initial comoving radius, $x_{\mathrm{i}}$, to a maximum radius, $x_{\mathrm{m}}$ (usually named turn-around radius $x_{\mathrm{ta}}$ ), and eventually collapses to a singularity (for details how the singularity can be eliminated, see Engineer et al. 2000; Shaw \& Mota 2008). A collapse to a point will never occur in practice, since dissipative physics and the process of violent relaxation will convert the kinetic energy of

\footnotetext{
${ }^{3}$ Radiation was neglected since the study considered only the post-recombination epoch.
} 
collapse into random motions, giving rise to a "virialized" structure (virialization occurs at $\left.t \approx 2 t_{\max }\right)$. Once a non-linear object is formed, it will continue to attract matter in its neighborhood and its mass will grow by accretion of new material, in the process of "secondary infall".

In the paper of Gunn \& Gott (1972), the authors were interested in the formulation of a theory of infall of matter into clusters of galaxies. The equations of dynamics of the structure written by them are relativistic (Eq. 7 of Gunn \& Gott 1972), but they continued the treatment in terms of Newtonian mechanics. In their treatment it was supposed that the structure collapsed radially and that non-radial motions were not present. Several following papers showed how to introduce non-radial motions, and angular momentum, L, (Ryden \& Gunn 1987; Hiotelis 2002; Le Delliou \& Henriksen 2003; Del Popolo 2009; Del Popolo \& Kroupa 2009; Zukin \& Bertshinger 2010) preserving spherical symmetry ${ }^{4}$. The equations of the SCM with angular momentum can be written as (e.g., Peebles 1993; Zukin \& Bertshinger 2010)

$$
\frac{d^{2} R}{d t^{2}}=-\frac{G M}{R^{2}}+\frac{L^{2}}{M^{2} R^{3}} .
$$

The SCM equations can be written in terms of the overdensity $\delta$, using General Relativity (Gatzanaga \& Lobo 2001) or in the Pseudo-Newtonian (PN) approach to cosmology (Lima et al. 1997). In the PN approach, the evolution equations of $\delta$ in the non-linear regime have been obtained and used in the framework of the spherical and ellipsoidal collapse and structure formation by several authors (Ohta et al. 2003; Ohta et al. 2004; Abramo et al. 2007).

In order to obtain the quoted equations, we assume that the fluid satisfies the equation-of-state $P=w \rho$ (here, we set the velocity of light $c=1$ ), and we use in the calculation the generalizations of the continuity equation, of Euler's equation (both valid for each fluid species $j$ ), and of Poisson's equation (which is valid for the sum of all fluids) given as (Lima et al. 1997; Abramo et al. 2007)

$$
\begin{gathered}
\frac{\partial \rho_{j}}{\partial t}+\vec{\nabla}_{\vec{r}} \cdot\left(\vec{u}_{j} \rho_{j}\right)+p_{j} \vec{\nabla}_{\vec{r}} \cdot \vec{u}_{j}=0, \\
\frac{\partial \vec{u}_{j}}{\partial t}+\left(\vec{u}_{j} \cdot \vec{\nabla}_{\vec{r}}\right) \vec{u}_{j}=-\vec{\nabla}_{\vec{r}} \Phi-\frac{\vec{\nabla}_{\vec{r}} p_{j}}{\rho_{j}+p_{j}}, \\
\nabla_{\vec{r}}^{2} \Phi=4 \pi G \sum_{k}\left(\rho_{k}+3 p_{k}\right),
\end{gathered}
$$

where $\rho_{j}, p_{j}, \vec{u}_{j}$ and $\Phi$ denote, respectively, the density, pressure, velocity and the Newtonian gravitational potential of the cosmic fluid.

This set of equations is valid only for subhorizon scales and they do not take into account possible effects at scales larger than the horizon. Despite their limitations, they proved to be very useful to describe structure formation in quintessence models, and their predictions were in agreement with the results of $N$-body simulations (see, for example, the appendix in Pace et al. (2010) and references therein). The equations, as presented here, are not simply a generalization of Newtonian

\footnotetext{
4 Spherical symmetry is preserved if one assumes that the distribution of angular momenta of particles is random, implying a net null mean angular momentum.
} 
hydrodynamical equations, but they have a well defined theoretical justification. As detailed in Pace et al. (2010), to which we refer for more details, the PN equations can be derived directly from General Relativity, by assuming the stressenergy tensor of a perfect fluid characterized by density and pressure. Given the stress-energy tensor $T^{\mu \nu}$, one computes its four-divergence $\left(\nabla_{\mu} T^{\mu \nu}=0\right)$ and its contraction with the projection tensor $h_{\alpha \mu}=g_{\alpha \mu}+u_{\alpha} u_{\mu}$. The first, contracted with the four-velocity, gives the general relativistic continuity equation, the second the Euler equation. Specifying the usual Newtonian metric and under the assumption of weak field and small velocities $(v \ll c)$ we obtain the PN equations.

We would like to recall that in this work we want to generalize the work of Fernandes et al. (2012) taking into account the contribution of the shear and rotation terms. We therefore closely follow the derivation of their equations.

As is customary, all quantities of interest are separated into a static component and a perturbation, as follows:

$$
\begin{aligned}
\rho(\vec{x}, t) & =\bar{\rho}(1+\delta(\vec{x}, t)), \\
P(\vec{x}, t) & =w \rho(\vec{x}, t) c^{2}, \\
\Phi(\vec{x}, t) & =\Phi_{0}(\vec{x}, t)+\phi(\vec{x}, t), \\
\vec{u}(\vec{x}, t) & =a[H(a) \vec{x}+\vec{v}(\vec{x}, t)],
\end{aligned}
$$

where $\vec{v}(\vec{x}, t)$ is the comoving peculiar velocity, and $H(a)$ is the Hubble function. Inserting Eqs. (8)-(11) into Eqs. (5)-(7) (see also Pace 2010), using comoving coordinates $\vec{x}=\vec{r} / a$, and considering that the equation of state $w_{j}$ and the effective sound speed $c_{\text {eff }, j}^{2}$ are functions of time only, the perturbed equations for the density contrast, $\delta_{j}=\delta \rho_{j} / \rho_{j}$, those for the peculiar velocity $v_{j}$ of fluid $j$, and that for the potential are given by

$$
\begin{gathered}
\dot{\delta}_{j}+3 H\left(c_{\mathrm{eff}, j}^{2}-w_{j}\right) \delta_{j}= \\
-\left[1+w_{j}+\left(1+c_{\mathrm{eff}, j}^{2}\right) \delta_{j}\right] \frac{\vec{\nabla} \cdot \vec{v}_{j}}{a}-\frac{\vec{v}_{j} \cdot \vec{\nabla} \delta_{j}}{a}, \\
\dot{\vec{v}}_{j}+H \vec{v}_{j}+\frac{\vec{v}_{j} \cdot \vec{\nabla}}{a} \vec{v}_{j}=-\frac{\vec{\nabla} \phi}{a}-\frac{c_{\mathrm{eff}, j}^{2} \vec{\nabla} \delta}{a\left[1+w_{j}+\left(1+c_{\mathrm{eff}, j}^{2}\right) \delta_{j}\right]}, \\
\frac{\nabla^{2} \phi}{a^{2}}=4 \pi G \sum_{k} \rho_{0_{k}} \delta_{k}\left(1+3 c_{\mathrm{eff}, k}^{2}\right),
\end{gathered}
$$

where $c_{\mathrm{eff}, j}^{2} \equiv \delta p_{j} / \delta \rho_{j}$ is the effective sound speed of each fluid. ${ }^{5}$

The previous equations can be simplified as in Abramo et al. (2007) and Fernandes et al. (2012),

$$
\begin{aligned}
\delta_{j}^{\prime}= & -\frac{3}{a}\left(c_{\mathrm{eff}, j}^{2}-w_{j}\right) \delta_{j} \\
& -\left[1+w_{j}+\left(1+c_{\mathrm{eff}, j}^{2}\right) \delta_{j}\right] \frac{\theta}{a^{2} H},
\end{aligned}
$$

${ }^{5}$ Note that in the previous equations, $\vec{\nabla}$ refers to gradient with respect to comoving coordinates $\vec{x}$. 


$$
\begin{aligned}
\theta^{\prime}= & -\frac{\theta}{a}-\frac{\theta^{2}}{3 a^{2} H} \\
& -\frac{3 H}{2} \sum_{j} \Omega_{j} \delta_{j}\left(1+3 c_{\mathrm{eff}, j}^{2}\right)-\frac{\sigma^{2}-\omega^{2}}{a^{2} H},
\end{aligned}
$$

where the prime denotes the derivative with respect to $a, \Omega_{j}=\left(8 \pi G / 3 H^{2}\right) \rho_{j}$, and where $\theta_{j} \equiv \nabla \cdot \vec{v}_{j}$ and $\vec{v}_{j}$ is the peculiar velocity field. As previously reported, in the former derivation it was assumed that $w_{j}$ and $c_{\mathrm{eff}, j}^{2}$ are functions of time only (see Fernandes et al. 2012; Abramo et al. 2007).

We remind the reader that shear and vorticity are present already in Eq. (13), via the term $(\vec{v} \cdot \vec{\nabla}) \vec{v}$. To obtain $\sigma$ and $\omega$, one simply needs to take the divergence of Eq. (13), and recall the decomposition

$$
\nabla \cdot\left[\left(\overrightarrow{u_{j}} \cdot \nabla\right) \overrightarrow{u_{j}}\right]=\frac{1}{3} \theta_{j}^{2}+\sigma^{2}-\omega^{2},
$$

where the expansion term, $\theta_{j}$, is given by $\theta=\nabla_{\vec{x}} \cdot \vec{u}$, the shear tensor, $\sigma$, is given by $\sigma^{2}=\sigma_{i j} \sigma^{i j}$ and the rotation tensor is given by $\omega^{2}=\omega_{i j} \omega^{i j}$.

At this point we want to stress that combining Eqs. (15) and (16) will lead to a quite complicated second order ordinary differential equation that generalizes the usual evolution of the perturbations. An easy way to recover it, instead of combining Eqs. (15) and (16), is the following.

As a first step, one takes the divergence of Euler's equation (Eq. 13), and using the decomposition given in Eq. (17), taking the time derivative of Eq. (12), and combining the previous three equations one gets

$$
\begin{gathered}
\ddot{\delta}_{j}+\left(2 H-\frac{\dot{w}_{j}}{1+w_{j}}\right) \dot{\delta}_{j}-\frac{4+3 w_{j}}{3\left(1+w_{j}\right)} \frac{{\dot{\delta_{j}}}^{2}}{1+\delta_{j}}- \\
4 \pi G \bar{\rho}\left(1+w_{j}\right)\left(1+\delta_{j}\right) \sum_{k} \rho_{k} \delta_{k}\left(1+3 w_{k}\right)- \\
\left(1+w_{j}\right)\left(1+\delta_{j}\right)\left(\sigma^{2}-\omega^{2}\right)=0,
\end{gathered}
$$

or, in terms of the scale factor,

$$
\begin{array}{r}
\delta_{j}^{\prime \prime}+\left(\frac{3}{a}+\frac{E^{\prime}}{E}-\frac{w_{j}^{\prime}}{1+w_{j}}\right) \delta_{j}^{\prime}-\frac{4+3 w_{j}}{3\left(1+w_{j}\right)} \frac{\delta_{j}^{\prime 2}}{1+\delta_{j}} \\
-\frac{3}{2} \frac{1}{a^{2} E^{2}(a)}\left(1+w_{j}\right)\left(1+\delta_{j}\right) \sum_{k} \Omega_{k, 0} g(a)\left(1+3 w_{k}\right) \delta_{k}- \\
\frac{1}{a^{2} E^{2}(a)}\left(1+w_{j}\right)\left(1+\delta_{j}\right)\left(\sigma^{2}-\Omega^{2}\right)=0,
\end{array}
$$

where $\bar{\rho}$ is the background mass density, $E(a)$ is the expansion function (see Pace et al. 2010), and $g(a)$ is a function specifying the time evolution of the dark-energy model considered (see Pace et al. 2010).

The two previous equations are equivalent to Eq. (10) and Eq. (12) in Sandvik et al. (2004), with the difference that in the present paper they are expressed in real space and not in Fourier space, and that they contain both $\sigma$ and $\omega$. 
It is also important to notice that our derivation is very general and it should not be considered as an expansion in the overdensity parameter. The equations obtained have very broad validity and they hold also in the case when $\delta \gg 1$.

Notice that the number of equations for $\delta_{j}$ is equal to the number of cosmological fluids in the system while we have only one equation for $\theta$. This is due to the fact that for a 'top-hat' profile, resulting in $\vec{\nabla} \delta_{j}=0$, the peculiar velocity is the same for all fluids $\left(\theta_{j} \equiv \theta, \sigma_{j} \equiv \sigma, \omega_{j} \equiv \omega\right)$, resulting in only one equation for $\theta$.

The evaluation of the term $\sigma^{2}-\omega^{2}$ was discussed in Del Popolo et al. (2013b,c) by defining the ratio $\beta$ between the rotational and gravitational terms in Eq. (4):

$$
\beta=\frac{L^{2}}{M^{3} R G}
$$

In the case of spiral galaxies like the Milky Way, $\beta \simeq 0.04$. Its value is larger for smaller size perturbations (such as of dwarf galaxy size) and smaller for larger size perturbations (for galaxy clusters, the ratio is of the order of $10^{-6}$ ). In order to obtain a value for $\delta_{\mathrm{c}}$ similar to the one obtained by Sheth et al. (2001) (see also Del Popolo 2006), we set $\beta=0.04$ for galactic masses (see also Del Popolo et al. 2013b,c).

In order to integrate Eq. (16) we need to make explicit the $\sigma^{2}-\omega^{2}$ term. This was done in Del Popolo et al. (2013b,c). Based on the above outlined argument for rotation, one may calculate the same ratio between the gravitational term and the extra term appearing in Eq. (21) thereby obtaining

$$
\frac{\sigma^{2}-\omega^{2}}{a^{2} H^{2}}=-\frac{3}{2} \beta \sum_{j} \Omega_{j} \delta_{j}\left(1+3 c_{\mathrm{eff}, j}^{2}\right) .
$$

In the following, we will consider $\beta=0.04$, corresponding to spiral galaxies similar to the Milky Way, and $\beta=0.02$ and $\beta=0.01$, corresponding to systems in which rotation is less important.

The term $c_{\text {eff }}^{2}$ used is the same as that proposed by Fernandes et al. (2012), namely,

$$
c_{\mathrm{eff}}^{2}==-\frac{C}{\rho^{1+\alpha}} \frac{(1+\delta)^{-\alpha}-1}{\delta}=w \frac{(1+\delta)^{-\alpha}-1}{\delta} .
$$

Eq. (24) shows that the effective sound speed depends on the collapsed region (through $\delta$ ) and the background (through $w$ ). The parameter $w$ relative to the collapsed region, namely $w_{\mathrm{c}}$, is given by Eq. (20) of Fernandes et al. (2012):

$$
w_{\mathrm{c}}=-\frac{C}{(\rho(1+\delta))^{1+\alpha}}=\frac{w}{(1+\delta)^{1+\alpha}} .
$$

In order to study the effect of $\alpha$ on the growth of perturbations, we solved a system of two Eqs. (15) (one for $\mathrm{gCg}$ and one for baryons), and one Eq. (16). We used three values of $\alpha$, namely, $\alpha=0$ (model equivalent to the $\Lambda \mathrm{CDM}$ ) and $\alpha=0.5,1$, and $\bar{C}=0.75$. As previously reported, we considered three values for 

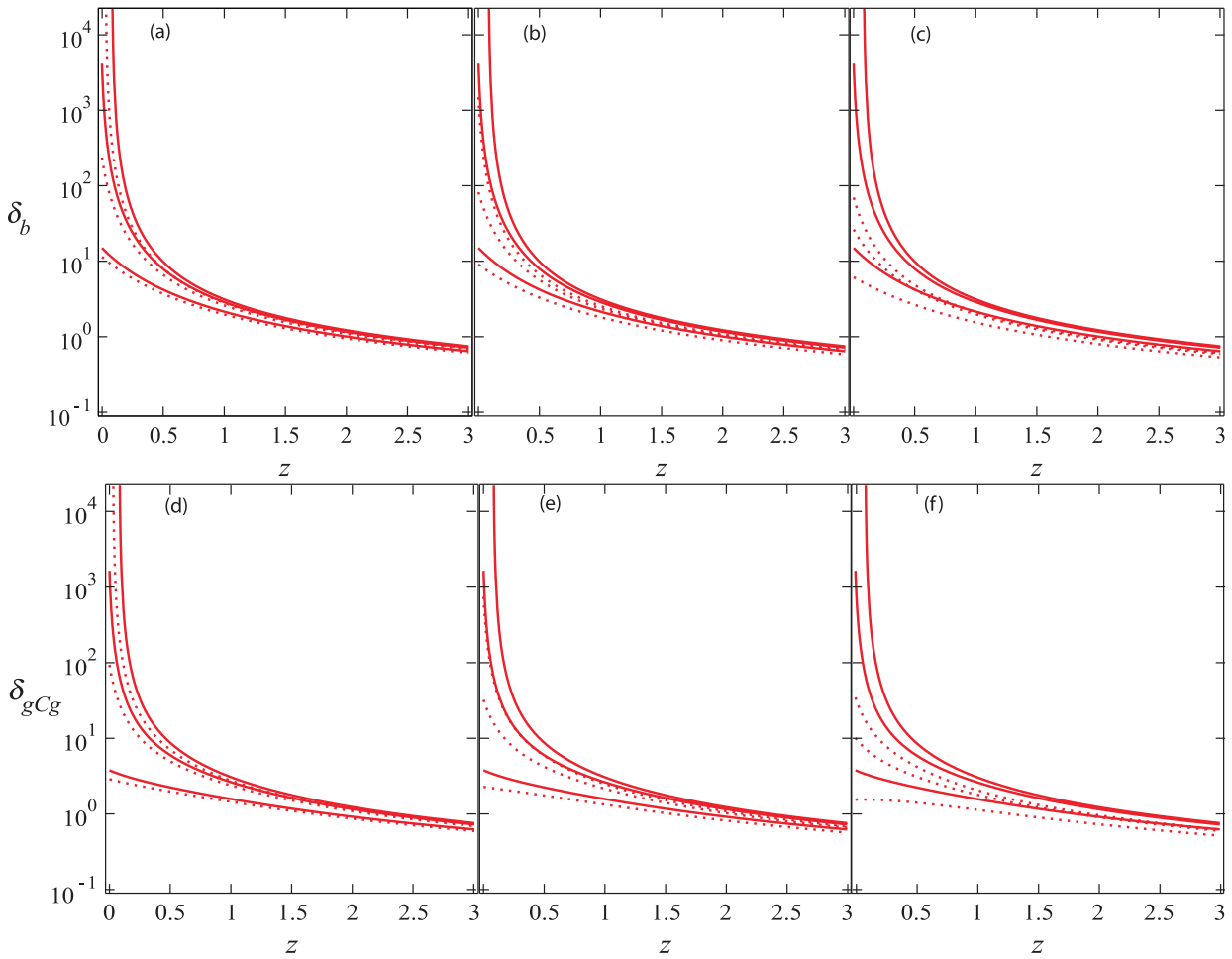

Fig. 1. Growth of perturbations for the SCM in gCg-dominated universes. Top (panels $\mathrm{a}-\mathrm{c}): \delta_{b}$ vs $z$. Bottom (panels $\left.\mathrm{d}-\mathrm{f}\right): \delta_{\mathrm{gCg}}$ vs $z$. Panels $(\mathrm{a}-\mathrm{c})$ : from top to bottom, the values of $\alpha$ are $1,0.5,0$. The solid line represents $\delta_{\mathrm{b}}$ without the effect of the additive term $\sigma^{2}-\omega^{2}$, whereas the dotted line is shown with its presence. The value of $\beta$ is 0.01 in panel (a), 0.02 in panel (b), and 0.04 in panel (c). Panels (d-f): similar to panels $(a-c)$ but for $\delta_{\mathrm{gCg}}$.

$\beta$, namely, $\beta=0.01,0.02$, and 0.04 . The initial conditions for the system and the values of the density parameters and Hubble constant are the same as in Fernandes et al. (2012), and in agreement with recent values for the $\Lambda$ CDM model (Komatsu et al. 2011). As in Fernandes et al. (2012), $p_{\mathrm{b}}=w_{\mathrm{b}}=c_{\mathrm{s}, \mathrm{b}}^{2}=c_{\mathrm{eff}, \mathrm{b}}^{2}=0$.

\section{RESULTS}

In our calculations, we used the same initial conditions for all of the models considered. In Figs. 1a-1c (1d-1f), the solid lines represent the evolution of $\delta_{\mathrm{b}}$ $\left(\delta_{\mathrm{gCg}}\right)$ in the case the term $\sigma^{2}-\omega^{2}$ is not present, similarly to Fernandes et al. $(2012)$, and from top to bottom of each plot the value of $\alpha$ changes, i.e., $\alpha=1$, $0.5,0$.

As noticed by Fernandes et al. (2012), larger values of $\alpha$ produce a faster collapse via larger values of the effective sound speed at lower $z$. The different behavior of $\delta_{\mathrm{b}}\left(\delta_{\mathrm{gCg}}\right)$ for different $\alpha$ reflects the different evolution of $c_{\mathrm{eff}}^{2}$ and $w$ on the equations of evolution of $\delta$. Moreover, at smaller $z$, when DE dominates, larger values of $\alpha$ produce a later transition from DM to DE dominated stages of the $\mathrm{gCg}$ universes. 

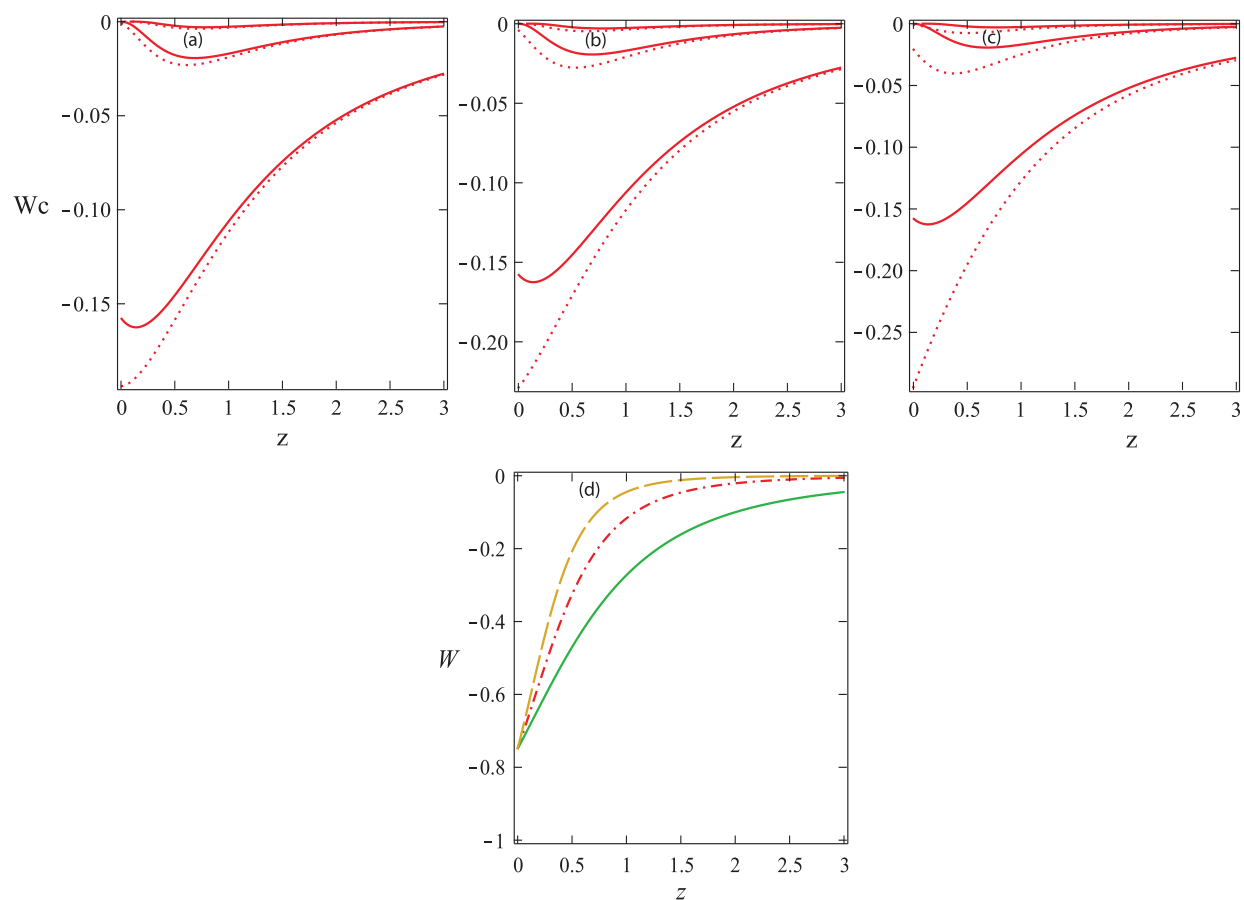

Fig. 2. Evolution of $w_{\mathrm{c}}$ and $w$ with $z$ for gCg universes. Panels $(\mathrm{a}-\mathrm{c})$ : from left to right, the values of $\alpha$ are $1,0.5,0$. The solid line represents $\delta_{\mathrm{b}}$ without the effect of the additive term $\sigma^{2}-\omega^{2}$, whereas the dotted line is shown with its presence. The value of $\beta$ is 0.01 in panel (a), 0.02 in panel (b), and 0.04 in panel (c). Panel (d): $w$ versus $z$ for $\alpha=1$ (dashed line), 0.5 (dot-dashed line), 0 (solid line).

The dotted line in Fig. 1 represents $\delta_{\mathrm{b}}\left(\delta_{\mathrm{gCg}}\right)$ for the same values of $\alpha$ but when $\sigma^{2}-\omega^{2}$ is different from zero. In Fig. 1a the value of $\beta$ is 0.01 , while in Figs. 1b and $1 \mathrm{c}$ it is 0.02 and 0.04 , respectively, and similarly in Figs. 1d-1f. The effect of $\sigma^{2}-\omega^{2}$ is that of slowing down the collapse, so that the collapse acceleration produced by larger values of $\alpha$ is mitigated by the additive term. Somehow, the presence of the additive term can be mimicked by a reduction of $\alpha$.

In Figs. 2a-2c, we calculate $w_{\mathrm{c}}$ using Eq. (25) and the previously calculated values of $\delta_{\mathrm{gCg}}$, while in Fig. 2d we calculate $w$. The solid lines in Figs. 2a-2d represent $w_{\mathrm{c}}$ when $\sigma^{2}-\omega^{2}$ is not taken into account, for $\alpha=1$ (top curve), 0.5 (median curve), 0 (bottom curve). As can be seen, $\alpha$ has a strong effect on the results. A larger $\alpha$ produces a faster collapse and $w_{\mathrm{c}}$ closer to zero, and, moreover, in a later transition from $\mathrm{DM}$ to $\mathrm{DE}$ dominated stages of the $\mathrm{gCg}$ universes. The quoted result is obtained for a fixed value of $\bar{C}$ (in our case, $\bar{C}=0.75$ ). If we increase the content of $\mathrm{DE}$ of the system, corresponding to increasing the value of $\bar{C}$, the collapse will happen at later times or it will be prevented with the consequence that $w_{\mathrm{c}}$ will no longer be close to zero. The dotted lines represent the same quantity when the additive term is taken into account. In Fig. 2a the value of $\beta$ is 0.01 , while in Figs. $2 \mathrm{~b}$ and $2 \mathrm{c}$ it is 0.02 and 0.04 , respectively. Since the effect of the additive term is to slow down the collapse, the effect on $w_{\mathrm{c}}$ is that of showing a more pronounced departure from zero. Fig. $2 \mathrm{~d}$ represents $w$ 


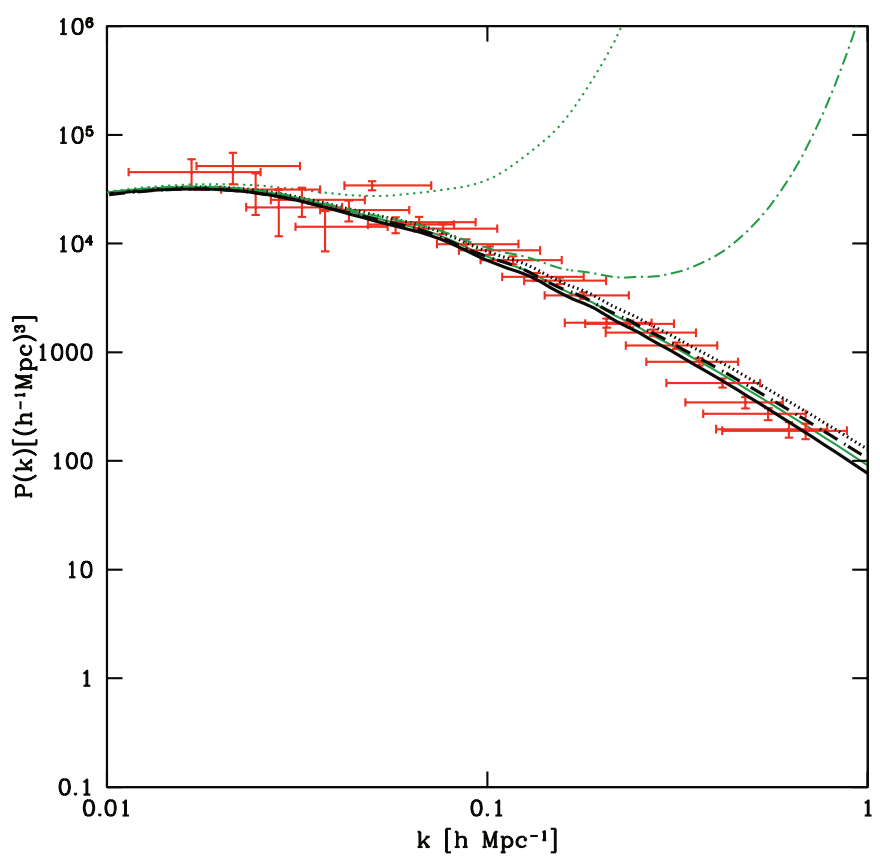

Fig. 3. UDM solution for perturbations as a function of the wavenumber $k$. The green dot, dot-dashed and solid curves are gCg models calculated by Sandvik et al. (2004), while the black curves are calculated in this paper with $\alpha=-10^{-4},-10^{-5}$ and $\alpha=0$, respectively. The data points are the power spectrum of the $2 \mathrm{df}$ galaxy redshift.

given by Eq. (3), depending on $\bar{C}, a$, and $\alpha$, and then independent of our additive parameter, and, consequently, identical to Fig. 2b of Fernandes et al. (2012). The solid line represents the case $\alpha=0$, the dash-dotted one the case $\alpha=0.5$, and the dashed line the case $\alpha=1$. The comparison of $w_{\mathrm{c}}$, a local (non-linear) parameter, with $w$, a global (linear) parameter, shows a clear evidence of the difference in the linear and non-linear dynamical behavior of the gCg.

The previous works (e.g., Sandvik et al. 2004) showed a problem in UDM models, namely, oscillations or exponential blowup of the dark matter power spectrum inconsistent with observations, the problem which is evident on galactic scales and only at recent times and that cannot be solved by taking baryons into account. Sandvik et al. (2004) showed that gravitational effects of DM, at late time, can add fluctuations to baryons but that they are unable to erase the ones already present.

Our result, concerning the effect of $\alpha$ on the growth of perturbations is partially in disagreement with the linear theory of perturbation in $\mathrm{gCg}$ universes (e.g., Sandvik et al. 2004) and in agreement with Fernandes et al. (2012) findings. In our study, however, due to the additive term which has its maximum effect on galactic scales, the growth of perturbation is slowed down. This somehow implies that the presence of additive term works towards reducing possible present oscillations as found by Sandvik et al. (2004).

In order to show this, we used the spectrum obtained by Sandvik et al. (2004), applied our Eq. (21) written in Fourier space, and used it to evolve the fluctuations 
in the range $0<z<100$. The result is plotted in Fig. 3. The lines are explained in the caption. The Sandvik et al. (2004) model, not taking account of baryons, shear and vorticity, presents large changes on small scales for small $\alpha \neq 0$, and is in agreement with data for $\alpha=0$. In the case of our $\mathrm{gCg}$, the discrepancies shown by Sandvik et al.'s model are not present, confirming, as discussed previously, that baryons and non-linear effects change the $\mathrm{gCg}$ behavior.

\section{CONCLUSIONS}

In the present paper, we used the SCM to study how perturbations evolve in $\mathrm{gCg}$ universes, taking into account the effect of shear and rotation. We used the same $c_{\text {eff }}^{2}$ of Fernandes et al. (2012), and, in agreement with their work, we found that larger values of the parameter $\alpha$ speed up the collapse, but the additive term $\sigma^{2}-\omega^{2}$ produces a slowing down of this acceleration, visible in the figures showing the evolution of $\delta_{\mathrm{b}}$ and $\delta_{\mathrm{gCg}}$ (Fig. 1), and $w_{\mathrm{c}}$ (Fig. 2). The comparison of $w_{\mathrm{c}}$, a local (non-linear) parameter, with $w$, a global (linear) parameter, shows clear evidence of the difference in the linear and non-linear dynamical behavior of the $\mathrm{gCg}$.

We also showed that the blow-up of the dark matter power spectrum observed by Sandvik et al. (2004) is no longer present when baryons and the effects of shear and rotation are taken into account.

Notwithstanding the fact that the SCM is usually a faithful technique to study gravitational collapse and structure formation, with its results comparable to those of simulations by Ascasibar et al. (2007), it would be worthwhile to check the results of this paper against SPH simulations, that would allow to take account of spatial pressure gradients. Moreover, more realistic profiles would improve our understanding of the local dynamics of $\mathrm{gCg}$ universes, and how the background dynamics is influenced by local non-linear inhomogeneities.

\section{REFERENCES}

Abramo L. R., Batista R. C., Liberato L., Rosenfeld R. 2007, Journal of Cosmology and Astroparticle Physics, 11, 12

Allen S. W., Rapetti D. A., Schmidt R. W. et al. 2008, MNRAS, 383, 879

Amanullah R., Lidman C., Rubin D. et al. 2010, ApJ, 716, 712

Amendola L., Finneli F., Burigana C., Carturan D. 2003, JCAP, 07, 005

Ascasibar Y., Hoffman Y., Gottlöber S. 2007, MNRAS, 376, 393

Astashenok A. V., Del Popolo A. 2012, Class. Quant. Grav., 29, 5014

Avelino P. P., Beça L. M., de Calvarho J. P., Martins C. J., Copeland E. J. 2004, Phys. Rev. D, 69, 041301

Balakin A. B., Pavón D., Schwarz D. J., Zimdahl W. 2003, New J. Phys., 5, 85

Basilakos S., Plionis M., Solà J. 2010, Phys. Rev. D, 82, 3512

Bertacca D., Bartolo N., Matarrese S. 2010, Adv. Astron., 2010, 78

Bertolami O. 2004, MNRAS, 353, 329

Bilic N., Lindebaum R. J., Tupper G. B., Viollier R. D. 2004, JCAP, 11, 008

Cardone V. F., Del Popolo A. 2012, MNRAS, 427, 3176

Cardone V. F., Del Popolo A., Tortora C., Napolitano N. R. 2011a, MNRAS, 416, 1822

Cardone V. F., Leubner M. P., Del Popolo A. 2011b, MNRAS, 414, 2265 
Chaplygin S. 1901, Sci. Mem. Moscow Univ., Math. Phys., 21, 1

Copeland E. J., Sami M., Tsujikawa S. 2006, Int. J. Mod. Phys. D, 15, 1753

Del Popolo A. 1999, A\&A, 344, 17

Del Popolo A. 2002, MNRAS, 336, 81

Del Popolo A. 2006, ApJ, 637, 12

Del Popolo A. 2007, Astron. Rep., 51, 169

Del Popolo A. 2009, ApJ, 698, 2093

Del Popolo A. 2010, MNRAS, 408, 1808

Del Popolo A. 2011, JCAP, 7, 014

Del Popolo A. 2012a, MNRAS, 419, 971

Del Popolo A. 2012b, MNRAS, 424, 38

Del Popolo A. 2013, AIP Conf. Proc., 1548, 2

Del Popolo A. 2014, IJMPD, 23, 1430005

Del Popolo A., Gambera M. 1996, A\&A, 308, 373

Del Popolo A., Gambera M. 1997, A\&A, 321, 691

Del Popolo A., Gambera M. 2000, A\&A, 357, 809

Del Popolo A., Hiotelis N. 2014, JCAP, 01, 047

Del Popolo A., Kroupa P. 2009, A\&A, 502, 733

Del Popolo A., Cardone V. F., Belvedere G. 2013a, MNRAS, 429, 1080

Del Popolo A., Pace F., Lima J. A. S. 2013b, MNRAS, 430, 628

Del Popolo A., Pace F., Lima J. A. S. 2013c, IJMPD, 22, 1350038

Del Popolo A., Lima J. A. S., Fabris J. C., Rodrigues D. C. 2014, JCAP, 04, 021

Dev A., Jain D., Alcaniz J. S. 2004, A\&A, 417, 847

Engineer S., Kanekar N., Padmanabhan T. 2000, MNRAS, 314, 279

Fernandes R. A. A., de Calvarho J. P. M., Kamenshchik A. Yu., Moschella U., da Silva A. 2012, Phys. Rev. D., 85, 083501

Frieman J. A., Turner M. S., Huterer D. 2008, ARA\&A, 46, 385

Gaztanaga E., Lobo J. A. 2001, ApJ, 548, 47

Gunn J. E., Gott J. R. 1972, ApJ, 176, 1

Hiotelis N., Del Popolo A. 2002, A\&A, 382, 84

Hiotelis N., Del Popolo A. 2006, Ap\&SS, 301, 67

Hiotelis N., Del Popolo A. 2013, MNRAS, 436, 163

Kamenshchik A., Moschella U., Pasquier V. 2001, Phys. Lett. B, 511, 265

Komatsu E., Smith K. M., Dunkley J. 2011, ApJS, 192, 18

Le Delliou M., Henriksen R. N. 2003, A\&A, 408, 27

Li M., Li X. D., Wang S., Wang Y. 2011, Commun. Theor. Phys., 56, 525

Lima J. A. S., Zanchin V., Brandenberger R. H. 1997, MNRAS, 291, L1

Navarro J. F., Ludlow A., Springel V. et al. 2010, MNRAS, 402, 21

Ohta Y., Kayo I., Taruya A. 2003, ApJ, 589, 1

Ohta Y., Kayo I., Taruya A. 2004, ApJ, 608, 647

Pace F., Waizmann J., Bartelmann M. 2010, MNRAS, 406, 1865

Peebles P. J. E. 1993, Principles of Physical Cosmology, Princeton University Press

Ryden B. S., Gunn J. E. 1987, ApJ, 318, 15

Sandvik H. B., Tegmark M., Zaldarriaga M., Waga I. 2004, Phys. Rev. D, 69, 123524

Shaw D. J., Mota D. F. 2008, ApJ, 174, 277 
Sheth R. K., Mo H. J., Tormen G. 2001, MNRAS, 323, 1

Spergel D. N., Bean R., Doré O. et al. 2007, ApJS, 170, 377

Weinberg S. 1989, Rev. Mod. Phys., 61, 1

Zhu Z.-H., Fujimoto M.-K., He X.-T. 2004, ApJ, 603, 365

Zukin P., Bertschinger E. 2010, Phys. Rev. D, 82, 104044 